\title{
On the Empirical Efficiency of the Vertex Contraction Algorithm for Detecting Negative Cost Cycles in Networks
}

\author{
K. Subramani and D. Desovski \\ LDCSEE, West Virginia University, \\ Morgantown, WV \\ $\{$ ksmani, desovski\}@csee.wvu.edu
}

\begin{abstract}
In this paper, we present a comprehensive empirical analysis of the Vertex Contraction (VC) algorithm for the problem of checking whether a directed graph with positive and negative costs on its edges has a negative cost cycle (NCCD). VC is a greedy algorithm, first presented in [SK05], for NCCD and is the only known greedy strategy for this problem. In SK05] we compared a naive implementation of VC with the "standard" Bellman-Ford (BF) algorithm for the same problem. We observed that our algorithm performed an order of magnitude better than the BF algorithm on a range of randomly generated inputs, thereby conclusively demonstrating the superiority of our approach. This paper continues the study of contrasting greedy and dynamic programming approaches, by comparing VC with a number of sophisticated implementations of the BF algorithm.
\end{abstract}

\section{Introduction}

This paper contrasts the performance of the Vertex Contraction (VC) algorithm with existing algorithms for the Negative Cost Cycle detection (NCCD) problem. NCCD is defined as follows: Given a directed graph $\mathbf{G}=<\mathbf{V}, \mathbf{E}, \mathbf{c}>$, where $\mathbf{V}=$ $\left\{v_{1}, v_{2}, v_{3}, \ldots, v_{n}\right\},|\mathbf{V}|=n, \mathbf{E}=\left\{e_{i j}: v_{i} \sim v_{j}\right\},|\mathbf{E}|=m$, and a cost function $\mathbf{c}: \mathbf{E} \rightarrow \mathbf{Z}$, is there a negative cost cycle in $\mathbf{G}$ ? There are no restrictions on the edge costs, i.e., they can be arbitrary integers as opposed to small integers, which is a requirement of scaling algorithms Gol95. We note that the problem, as specified, is a decision problem, in that all that is asked of an algorithm is to detect the presence of a negative cycle.

Some of the important application areas of NCCD include Image Segmentation, Temporal Constraint Solving, scheduling and System Verification [SK05]. Algorithms for negative cost cycle detection can be broadly classified as comparison based or scaling based. Comparison based algorithms in turn are based on heuristics to efficiently solve the linear programming formulation of the shortest path problem as a min-cost flow problem [CG96, CGR96]. The VC algorithm is a comparison based algorithm which is different from all existing approaches to NCCD in that it is a purely local, greedy approach. 
Our work in this paper is motivated primarily by the need for a simple algorithm, with good performance characteristics. Whereas the naive BellmanFord approach for NCCD is admittedly simple, it suffers from significant performance drawbacks that are not explained using asymptotic analysis [SK05]. On the other hand, the techniques used to enhance the performance of BF as outlined in Gol95, CG96 and AMO93, suffer from the drawbacks of implementational difficulty and application specificity. We are therefore interested in an easy-to-implement comparison-based algorithm which can be modified in simple ways to provide reasonable performance on a wide variety of graphs.

\section{The Vertex Contraction Algorithm}

The vertex contraction procedure consists of eliminating a vertex from the input graph, by merging all its incoming and outgoing edges. Consider a vertex $v_{i}$ with incoming edge $e_{k i}$ and outgoing edge $e_{i j}$. When $v_{i}$ is contracted, $e_{k i}$ and $e_{i j}$ are deleted and a single edge $e_{k j}^{\prime}$ is added with cost $c_{k i}+c_{i j}$. This process is repeated for each pair of incoming and outgoing edges. Consider the edge $e_{k j}^{\prime}$ that is created by the contraction; it falls into one of the following categories:

(a) It is the first edge between vertex $v_{k}$ and $v_{j}$. In this case, nothing more is to be done.

(b) An edge $e_{k j}$ already existed between $v_{k}$ and $v_{j}$, prior to the contraction of $v_{i}$. In this case, if $c_{k j}^{\prime}<c_{k j}$, keep the new edge and delete the previously existing edge (since it is redundant); otherwise delete the new edge (since it is redundant).

Algorithm (2.1) is a formal description of our technique. The correctness and analysis of our technique can be found in SK05].

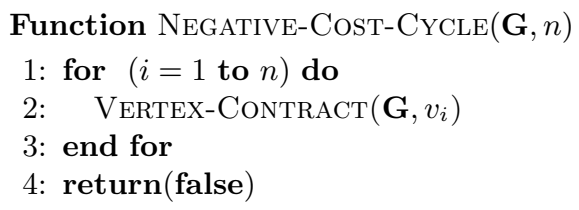

Algorithm 2.1. Negative cost cycle detection

\subsection{The Cruel Adversary}

The simple Vertex Contraction algorithm always chooses the next vertex to be contracted in a well-defined order; it is well-known that such a selection is susceptible to attack by a malicious adversary. For instance, an adversary could provide the graph in Figure (1) as input.

The above graph is sparse and has exactly $2 \cdot(n-1)$ edges. Observe that if vertex $v_{n}$ is contracted first, the resultant graph is the complete graph on 


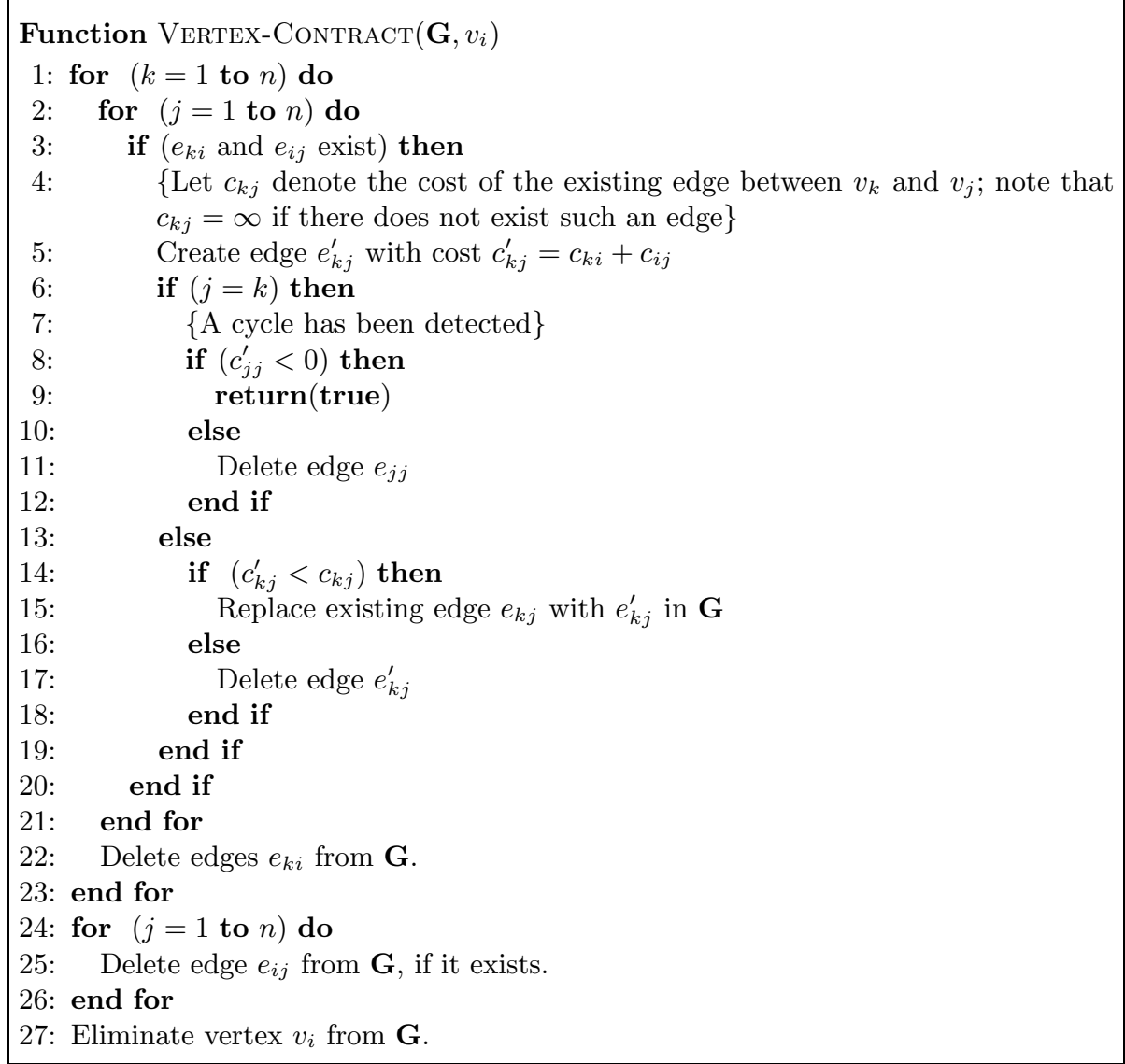

Algorithm 2.2. Vertex Contraction

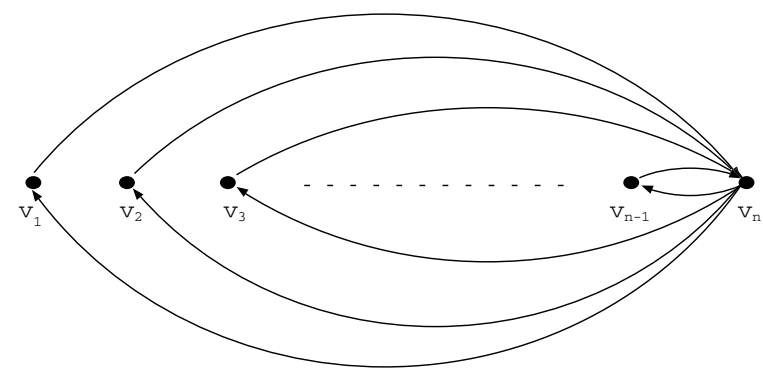

Fig. 1. Sparse graph that becomes dense after vertex contraction

$n-1$ vertices and therefore dense. We call this graph the cruel adversary; in our experiments, we made it a point to test the performance of all algorithms on this input. We could however choose the vertex to be contracted at random, 


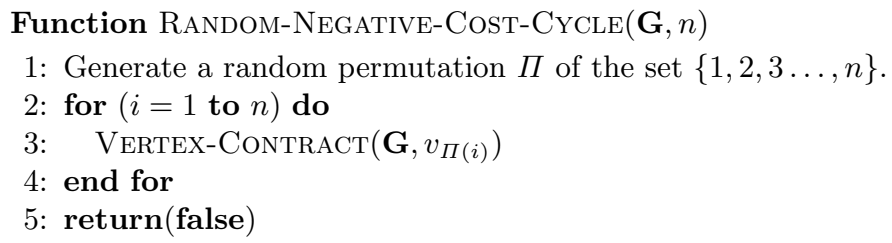

Algorithm 2.3. Random negative cost cycle detection algorithm

without affecting the correctness of the algorithm. We have implemented the Vertex Contraction algorithm with three different strategies based on how the vertices to be contracted are chosen:

(i) the vertex to be contracted is chosen in a well-defined order (VC),

(ii) the vertex to be contracted is the vertex with the smallest degree product (the degree product is calculated by multiplying the number of edges coming into a vertex $v_{i}$ with the number of edges going out of the vertex); this is accomplished by using a heap (HVC),

(iii) the vertex to be contracted is chosen at random (RVC). Algorithm (2.3) is a formal description of the random vertex contraction algorithm.

The experimental results on Cruel Adversary graphs have been relegated to the journal version of this paper. The extended version also contains a detailed description of the related work in the literature.

\section{$3 \quad$ Implementation}

Our experiments are classified into various categories, based on the following criteria:

(i) Type of input graph - Sparse with many small negative cycles (Type A), Sparse with a few long negative cycles (Type B), Dense with many small negative cycles (Type C), Dense with a few long negative cycles (Type D), the Cruel Adversary (Type E).

(ii) Type of Algorithm - Simple Vertex Contraction (VC), Vertex Contraction using a heap (HVC), Random Vertex Contraction (RVC), BellmanFord using a FIFO queue (BFFI), Bellman-Ford using a predecessor array (BFPR), Bellman-Ford using both a FIFO queue and a predecessor array (BFFP), Bellman-Ford using subtree disassembly (BFCT), and GoldbergRadzik (GORC).

(iii) Type of Graph Data Structure - Simple Pointer or Advanced Pointers.

\subsection{Implementation Details}

Two different types of graph data structures were used for the experiments viz., a simple pointer structure and an advanced pointer structure. Both structures require linear space. 
The simple pointer structure is also known as the adjacency-list representation CLR92. This representation makes use of an array of $n$ lists, one for each of the $n$ vertices of the graph. For each vertex $v_{i}$, we store the in-degree $d_{i}^{i n}$ (the number of edges going into the vertex), the out-degree $d_{i}^{\text {out }}$ (the number of edges going out of the vertex), and a singly linked list of edges going out from the vertex along with their weights. The linked lists of each vertex are sorted based on the destination vertex of the edge. Assuming that we are contracting vertex $v_{i}$, the vertex contraction operation for the simple pointer structure is performed as follows.

The time required to contract vertex $v_{i}$ by the simple pointer implementation is: $O\left(m+d_{i}^{\text {in }} \cdot d_{i}^{\text {out }}+\sum_{x}^{\left(v_{x}, v_{i}\right) \in E} d_{x}^{\text {out }}\right)$. In the worst case when the graph is dense $m=O\left(n^{2}\right), d_{i}^{\text {in }}=d_{i}^{\text {out }}=O(n)$, and the time complexity of the vertex contraction operation is $O\left(n^{2}\right)$.

In order to decrease the amount of time taken by the simple pointer structure, we consider the advanced pointer structure. For each vertex $v_{i}$, we store the indegree $d_{i}^{\text {in }}$, the out-degree $d_{i}^{\text {out }}$, and two doubly linked lists representing the edges. Each vertex has an out list, for the edges going out of the vertex, and an in list, for the edges going into the vertex. The out lists of each vertex are sorted based on the destination vertex of the edge. Assuming that we are contracting vertex $v_{i}$, the vertex contraction operation for advanced pointers is performed as follows. A variation of the advanced pointer structure in which the in-lists and out-lists are not sorted, is discussed in [MN99].

Table 1. Time required to perform vertex contraction using simple pointers

\begin{tabular}{|l|c|}
\hline \multicolumn{1}{|c|}{ Step } & Time to Execute \\
\hline 1) Find edges with destination vertex $v_{i}:\left(v_{x}, v_{i}\right)$ & $O(m)$ \\
2) For every edge $\left(v_{x}, v_{i}\right)$ found: & $O\left(d_{i}^{\text {in }}\right)$ \\
2-1) Remove the edge from the adjacency list of vertex $v_{x}$ & $O(1)$ \\
2-2) Merge $v_{i}$ 's list with $v_{x}$ 's list by adding those edges & \\
which are not present in $v_{x}$ 's list and updating those & $O\left(d_{i}^{\text {out }}+d_{x}^{\text {out }}\right)$ \\
who are already there & $O(1)$ \\
2-3) If a negative cost edge $\left(v_{x}, v_{x}\right)$ is created, the algorithm & \\
terminates with the negative cycle being detected & \\
\hline
\end{tabular}

Table 2. Time required to perform vertex contraction using advanced pointers

\begin{tabular}{|c|c|}
\hline Step & Time to Execute \\
\hline $\begin{array}{l}\text { 1) For every edge }\left(v_{x}, v_{i}\right) \text { in } v_{i} \text { 's in list } \\
\text { 1-1) Merge } v_{i} \text { 's out list with } v_{x} \text { 's out list } \\
\text { by adding those edges which are not present in } v_{x} \text { 's out list } \\
\text { and updating those who are already there, and also } \\
\text { updating the } i n \text { lists of the vertices appropriately } \\
\text { 1-2) If a negative cost edge }\left(v_{x}, v_{x}\right) \text { is created, the algorithm } \\
\text { terminates with the negative cycle being detected }\end{array}$ & $\begin{array}{c}O\left(d_{i}^{\text {in }}\right) \\
O\left(d_{i}^{\text {out }}+d_{x}^{\text {out }}\right)\end{array}$ \\
\hline
\end{tabular}


The time required to contract vertex $v_{i}$ by the advanced pointer implementation is: $O\left(d_{i}^{\text {in }} \cdot d_{i}^{\text {out }}+\sum_{x}^{\left(v_{x}, v_{i}\right) \in E} d_{x}^{\text {out }}\right)$. In the event that there exists some constant $c$ such that, for all $v_{x}, d_{x}^{\text {out }} \leq c \cdot d_{i}^{\text {out }}$, then this time bound simplifies to: $O\left(d_{i}^{\text {in }} \cdot d_{i}^{\text {out }}\right)$. In the worst case when $d_{i}^{\text {in }}=d_{i}^{\text {out }}=O(n)$ and hence the time complexity of the vertex contraction operation is $O\left(n^{2}\right)$.

\subsection{Implementation Remarks}

Note that

(i) For each experimental suite, we have provided only the graphical picture in this paper; the numerical tables are provided in the journal version of this paper. Our goal is to show that minor modifications to the VC framework result in significant performance enhancements, making its performance comparable to that of more sophisticated algorithms. Depending on the type of graph under consideration, a particular modification of $\mathrm{VC}$ may not be run.

(ii) All performance graphs have been drawn according to the following scale:

(a) On the $x$-axis, we represent the logarithm to the base 2 , of the number of vertices.

(b) On the $y$-axis, we represent the logarithm to the base 2 , of the running time in seconds.

\subsection{Experimental Setup for Sparse Graphs}

Sparse graphs were generated using the generator developed by Andrew Goldberg [CG96, which generates multiple edges between two vertices. Sparse graphs are defined as graphs with $o(n \cdot \log n)$ edges. We generated each graph 5 times using 5 different seeds for the random number generator. The times recorded are the medians over 5 executions of each implementation. (We used the median statistic based on related work in the literature; we have also maintained the worst-case times of each run. The timing profiles of the worst-case times and medians were very similar.)

Graphs of Type A and B were tested, with a number of vertices ranging from 500 to 10,000 in increments of 500 .

We define a small negative cycle as one consisting of at most $\frac{n}{100}$ vertices. We define a long negative cycle as one consisting of $\Omega\left(\frac{n}{2}\right)$ vertices. The number of long negative cycles in the input graphs was set to 4 .

\subsection{Experimental Results for Sparse Graphs}

It is easy to see from Figure (2) and Figure (3) that GORC outperforms all other implementations; this is true for both types of sparse graphs that were tested. $\mathrm{BFCT}$ and BFFP are comparable to GORC on most instances. AVC, and AHVC are far superior to BFFI and BFPR; they also outperform HVC and RVC on most instances. Bellman Ford using a FIFO queue (BFFI) and Bellman Ford 


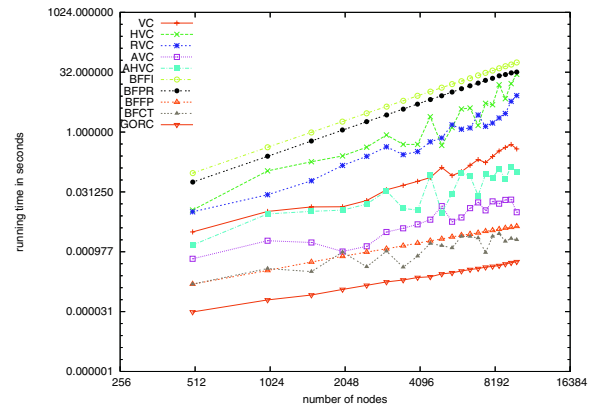

Fig. 2. Implementation execution times required to solve the Negative Cost Cycle detection problem for Type A graphs

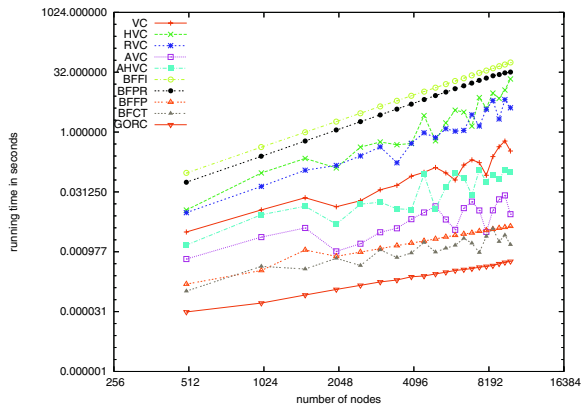

Fig. 3. Implementation execution times required to solve the Negative Cost Cycle detection problem for Type B graphs

using a predecessor array (BFPR) have been omitted from the graph, since the times for these two algorithms are much worse than any of the other algorithms tested.

\subsection{Experimental Setup for Dense Graphs}

Dense graphs were generated using the generator developed by Andrew Goldberg CG96], which generates multiple edges between two vertices. Dense graphs are defined as those graphs with $\Omega\left(\frac{n^{2}}{8}\right)$ edges. We generated each graph 5 times using 5 different seeds for the random number generator. The times recorded are the medians over 5 executions of each implementation.

Graphs of Type C and D were tested, with a number of vertices ranging from 500 to 10,000 in increments of 500, with small negative cycles and long negative cycles defined as in Section $\$ 3.3$. As mentioned before, HVC and AHVC were

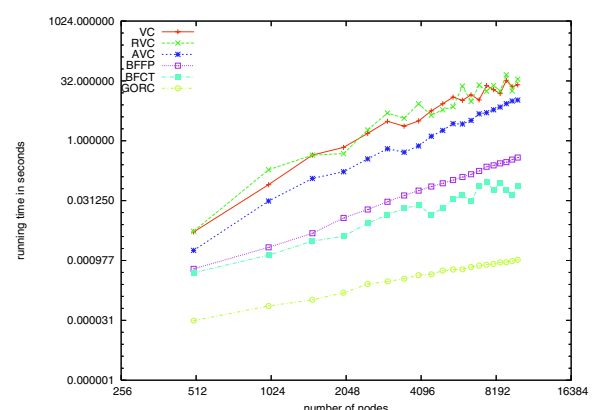

Fig. 4. Implementation execution times required to solve the Negative Cost Cycle detection problem for Type C graphs

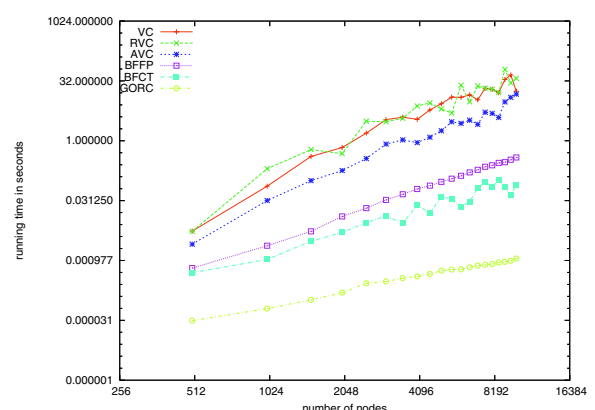

Fig. 5. Implementation execution times required to solve the Negative Cost Cycle detection problem for Type D graphs 
not run on dense graphs, since we found that there was no gain in terms of performance for the additional complexity in our test cases.

\subsection{Experimental Results for Dense Graphs}

It is easy to see from Figure (44) and Figure (5), that GORC outperforms all other implementations; this is true for both the types of dense graphs that were tested. BFCT performs slightly better than BFFP, although, both are comparable to GORC. AVC performs the best among the Vertex Contraction algorithms; although AVC, VC, and RVC are all far superior to BFFI and BFPR. Bellman Ford using a FIFO queue (BFFI) and Bellman Ford using a predecessor array (BFPR) have been omitted from the graph, since the times for these two algorithms are much worse than any of the other algorithms tested.

\section{References}

[AMO93] R. K. Ahuja, T. L. Magnanti, and J. B. Orlin. Network Flows: Theory, Algorithms and Applications. Prentice-Hall, 1993.

[CG96] Boris V. Cherkassky and Andrew V. Goldberg. Negative-cycle detection algorithms. In Josep Díaz and Maria Serna, editors, Algorithms-ESA '96, Fourth Annual European Symposium, volume 1136 of Lecture Notes in Computer Science, pages 349-363, Barcelona, Spain, 1996. Springer.

[CGR96] Boris V. Cherkassky, Andrew V. Goldberg, and T. Radzik. Shortest paths algorithms: Theory and experimental evaluation. Mathematical Programming, 73:129-174, 1996.

[CLR92] T. H. Cormen, C. E. Leiserson, and R. L. Rivest. Introduction to Algorithms. MIT Press and McGraw-Hill Book Company, Boston, Massachusetts, 2nd edition, 1992.

[Gol95] Andrew V. Goldberg. Scaling algorithms for the shortest paths problem. SIAM Journal on Computing, 24(3):494-504, June 1995.

[MN99] K. Mehlhorn and St. Näher. The LEDA Platform of Combinatorial and Geometric Computing. Cambridge University Press, Cambridge, 1999.

[SK05] K. Subramani and L. Kovalchick. A greedy strategy for detecting negative cost cycles in networks. Future Generation Computer Systems, 2005. Accepted, In Press. 